\title{
Factors influencing growth performance and estimation of genetic parameters in crossbred pigs
}

\author{
R. Ganesan ${ }^{1 *}$, P. Dhanavanthan ${ }^{1}$, Kiruthika ${ }^{1}$, P. Kumarasamy ${ }^{3}$, M. Murugan ${ }^{2}$ \\ and S. Jaishankar ${ }^{2}$ \\ ${ }^{1}$ Department of Statistics, Pondicherry University, Puducherry-605 014, India \\ ${ }^{2}$ Post Graduate Research Institute in Animal Sciences, Tamil Nadu Veterinary and Animal Sciences University, \\ Kattupakkam-603 203, Tamilnadu, India \\ ${ }^{3}$ Madras Veterinary College, Chennai - 600 007, Tamilnadu, India
}

\begin{abstract}
Piggery is an important livelihood for a large number of resource-poor rural households in India. Growth is an important trait of interest in any meat producing animal and body weight $(B W)$ measurements are good indicators of growth rate. The present study focuses on identifying the factors influencing pre and post weaning $B W$ and average daily gains $(A D G)$ in Large White Yorkshire-Desi crossbred pigs $(50 \%$ and $75 \%)$ using weekly $B W$ measurements from birth to 8 weeks of age and monthly $B W$ from $3^{\text {rd }}$ to $9^{\text {th }}$ month from 1,405 piglets born during the years 2002-2011. The overall least squares mean BW (kgs) at birth, $1^{\text {st }}, 2^{\text {nd }}, 3^{\text {rd }}, 4^{\text {th }}, 5^{\text {th }}$, $6^{t h}, 7^{\text {th }}, 8^{\text {th }}, 12^{\text {th }}, 16^{\text {th }}, 20^{\text {th }}, 24^{\text {th }}, 28^{\text {th }}, 32^{\text {nd }}$ and $36^{\text {th }}$ week were $1.090,1.93,2.76,3.76,4.518,5.461,6.591$, $7.584,8.670,12.959,18.211,24.254,34.052,41.970,48.414$ and 59.889 respectively. The overall least squares mean ADG (kgs) during 0-1, 1-2, 2- 3, 3-4, 4-5, 5-6, 6-7, 7-8, 0-8, 8-12, 12-16, 16-20, 20-24, 24-28, 28-32, 32$36,8-36$ weeks were $0.123,0.118,0.117,0.137,0.138,0.152,0.155,0.191,0.135,0.119,0.164,0.180,0.318$, $0.249,0.201,0.272$ and 0.255 respectively. Heritability estimated using full-sib analysis for $B W$ at various ages varied from 0.577 to 0.966. Estimated genetic correlations among pre-weaning $B W$ were positive and low in magnitude whilst among post-weaning $B W$, genetic correlations were high positive. The findings suggest that $12^{\text {th }}$ week $B W$ can be used for selecting increased $B W$ at $36^{\text {th }}$ week. The findings indicate scope for improvement through selection.
\end{abstract}

Keywords: Average daily gain, body weight, crossbred pigs, genetic correlation, heritability

\section{Introduction}

Exotic-desi crossbred pigs are rich source of animal protein. Bodyweights (BW) and growth rates are important indicators for animal breeders for evolving strategies for higher productivity. The pre and post weaning BW and growth rates in pigs are influenced by various genetic and non-genetic factors such as genetic group, sex, parity, litter size at birth, season and period of birth. Genetic analysis of BW through heritability and genetic correlation in crossbred pigs at different ages is essential to find out the scope for increasing the BW during slaughter age of pig through indirect selection and thus maximizing the profit of pig farms. Heritability estimates of growth traits establish the genetic potential of a herd and helps in its possible exploitation through selection [1]. In this work, an attempt was made to study the effect of various genetic and non-genetic factors affecting pre-weaning and post-weaning body weights and average daily weight gains (ADG) in half-bred $(50 \% \mathrm{CB})$ and graded progenies $(75 \% \mathrm{CB})$ of Large White Yorkshire (LWY) and Desi pigs. The genetic parameters, namely heritability, genetic correlations and phenotypic correlations have also been computed.

\subsection{Data description}

\section{Materials and Methods}

Data pertaining to 1,405 piglets belonging to two genetic groups i.e. $50 \%$ and $75 \%$ LWY - Desi crossbreds born during the years 2002-2011 were used in the present investigation. Piglets were weaned at 8 weeks of age. The data on year of birth were grouped into two periods, viz. period 1 (2002-2005) and period 2 (2006-2011). Month of birth was assigned to one of the four seasons viz. summer (Mar-May), pre-monsoon (Jun-Aug), monsoon (Sep-Nov) and winter (Dec-Feb). The litter size at birth (LSB) was classified into 4 groups viz. $1-3,4-6,7-9,10+$.

\subsection{Preliminary environment effects analysis}

The effects of genetic and non-genetic factors viz. genetic group, sex, parity, liter size at birth, season and period of birth on pre and post weaning growth traits were studied by least squares method followed by pair wise comparison of means. General linear model procedure was used to identify the effect of genetic and non- 
genetic factors on the observed body weights recorded at birth, 1, 2, 3, 4, 5, 6, 7, 8, 12, 16, 20, 24, 28, 32 and 36 weeks of age. The fixed model

$$
Y_{i j k l m n o}=\mu+G_{i}+S_{j}+L S_{k}+P_{l}+S B_{m}+P B_{n}+\varepsilon_{i j k l m n o}
$$

was used where $Y_{i j k l m n o}$ is the adjusted BW of the $o^{\text {th }}$ piglet, $\mu$ is the overall mean, $G_{i}$ is the fixed effect of the $i^{\text {th }}$ genetic group $(i=50 \% \mathrm{CB}, 75 \% \mathrm{CB}), S_{j}$ is the $j^{\text {th }}$ gender $(j=$ male, female $), L S_{k}$ is the $k^{\text {th }}$ litter size at birth group $(\mathrm{k}=1-3,4-6,7-9,10+), P_{l}$ is the $l^{\text {th }}$ Parity $(l=1,2+), S B_{m}$ is the $m^{\text {th }}$ season of birth ( $m=$ summer, pre-monsoon, monsoon, winter), $P B_{n}$ is the $n^{\text {th }}$ period of birth $(n=2002-05,2006-11)$ and $\varepsilon_{i j k l m o}$ is the error attributed to the $o^{\text {th }}$ piglet.

\subsection{Estimation of genetic parameters}

The scope for genetic improvement in an economically important trait of pig depends on its magnitude of heritability and genetic variance. Heritability, denoted by $h^{2}$, is one of the most important concepts in animal breeding as it is used to help plan breeding programs, determine management strategies, estimate breeding values of individual animals, and predict response to selection. It is a measure of the degree (0 to 1$)$ to which offspring resemble their parents for a specific trait and indicates how much confidence to place in the phenotypic performance of an animal when choosing parents of the next generation. For highly heritable traits where $h^{2}$ exceeds 0.40 , the animal's phenotype is a good indicator of genetic merit or breeding value. If however, $h^{2}$ is estimated low, the trait under study can only be improved by better feeding and management rather than by selection. Genetic correlation which studies the association between two traits due to additive gene effect, tells us how pairs of traits "co-vary" or change together. When genetic correlation is different from 0 , then more of the same genes affect both traits. Selection for one trait will increase/decrease the other depending on whether the genetic correlation is positive or negative. The phenotypic covariance is the sum of genetic and environmental covariances.

In species like pig, each male is mated to several females and each mating produces several offspring (multiple births) resulting in larger full sib families. Hence, full-sib method is used for the computation of genetic parameters in pigs. The observation $Y_{i j k}$ on the $k^{\text {th }}$ progeny from $j^{\text {th }}$ dam mated to $i^{\text {th }}$ sire is denoted by the following statistical model $Y_{i j k}=\mu+s_{i}+d_{i j}+e_{i j k}$, where $\mu$ is the population mean, $s_{i}$ is the effect of $i^{t h}$ sire, $d_{i j}$ is the effect of the $j^{\text {th }}$ dam mated to $i^{\text {th }}$ sire and $\mathrm{e}_{\mathrm{ijk}}$ is the environmental deviations attributed to individuals due to uncontrolled environmental and genetic factors. All the effects are assumed to be random, normal and independent with $E\left(s_{i}\right)=0, E\left(d_{i j}\right)=0, E\left(e_{i j k}\right)=0$ and $E\left(s_{i}^{2}\right)=\sigma_{s}^{2}, E\left(d_{i j}^{2}\right)=\sigma_{D}^{2}, E\left(e_{i j k}^{2}\right)=\sigma_{e}^{2}$.

The estimate of heritability from the full-sib analysis using the sire and dam component can be obtained as $h_{S+D}^{2}=\frac{2\left(\hat{\sigma}_{s}^{2}+\hat{\sigma}_{D}^{2}\right)}{\hat{\sigma}_{P}^{2}}$ where $\hat{\sigma}_{P}^{2}=\hat{\sigma}_{S}^{2}+\hat{\sigma}_{D}^{2}+\hat{\sigma}_{e}^{2}$ is the estimated total phenotypic variance, $\hat{\sigma}_{s}^{2}$ is the additive genetic variance due to sire, $\hat{\sigma}_{D}^{2}$ is the additive genetic variance due to dam and $\hat{\sigma}_{e}^{2}$ is the environmental variance. Standard error of heritability was approximated using the formula due to [2] and is given by $S E\left(h_{S+D}^{2}\right)=\frac{16 h_{S+D}^{2}}{T}$, where $\mathrm{T}=\mathrm{n} * \mathrm{~N}, \mathrm{n}$ is the average half sib family size and $\mathrm{N}$ is the total half sib families. The formula for genetic correlation between two traits $\mathrm{x}$ and $\mathrm{y}$ and its SE are given by $r_{g}=\frac{\operatorname{cov}_{s}(x y)}{\sqrt{\sigma_{s(x)}^{2} \sigma_{s(y)}^{2}}}$ and $S E\left(r_{g}\right)=\frac{1-r_{g}^{2}}{\sqrt{2}} \sqrt{\frac{S E\left(h_{x}^{2}\right) S E\left(h_{y}^{2}\right)}{h_{x}^{2} h_{y}^{2}}}$ [3]. The formula for phenotypic correlation between two traits $\mathrm{x}$ and $\mathrm{y}$ is $r_{p}=\frac{\operatorname{cov}_{p}(x y)}{\sqrt{\sigma_{p(x)}^{2} \sigma_{p(y)}^{2}}}=\frac{\operatorname{cov}_{g}(x y)+\operatorname{cov}_{e}(x y)}{\sqrt{\left(\sigma_{g(x)}^{2}+\sigma_{e(x)}^{2}\right)\left(\sigma_{g(x)}^{2}+\sigma_{e(y)}^{2}\right)}}$

Body weights recorded at birth, $1^{\text {st }}, 2^{\text {nd }}, 3^{\text {rd }}, 4^{\text {th }}, 5^{\text {th }}, 6^{\text {th }}, 7^{\text {th }}, 8^{\text {th }}, 12^{\text {th }}, 16^{\text {th }}, 20^{\text {th }}, 24^{\text {th }}, 28^{\text {th }}, 32^{\text {nd }}$ and $36^{\text {th }}$ week and the Average Daily Gains (ADG in gms) during $0-1,1-2,2-3,3-4,4-5,5-6,6-7,7-8,0-8,8-12,12-16$, 16-20, 20-24, 24-28, 28-32, 32-36, 8-36 weeks were used to estimate heritability and genetic correlation. The variance components used for the estimation of heritability and genetic correlation of each trait were obtained by Variance Components Procedure of SPSS software [4] using Restricted Maximum Likelihood (REML) method after adjusting for significant fixed effects. 


\section{Results and Discussion}

Out of a total of 1405 piglets used for the study, $27.3 \%$ belong to $50 \% \mathrm{CB}$ and $72.7 \%$ belong to $75 \% \mathrm{CB}$. There were $54.7 \%$ males and the rest females. The litter size at birth for $50 \% \mathrm{CB}$ varied from 1 to 11 with a mean of 6.50 while that of $75 \% \mathrm{CB}$ varied from 1 to 15 with a mean of 8.34 . The distribution of piglets in the various litter size groups were $5 \%(1-3), 24.9 \%(4-6), 47.6 \%(7-9)$ and $22.5 \%(10+)$. While $34.4 \%$ and $42.8 \%$ of piglets were born during summer and monsoon season, $19.7 \%$ and $3.0 \%$ were born during premonsoon and winter seasons. The pattern of birth during different seasons was similar for both the genetic groups. With regard to period of birth, $46.5 \%$ of animals were born during $2002-05$ while the rest $53.5 \%$ were born during $2006-11$. There were $64.2 \%$ of piglets belonging to parity 1 while the rest $35.8 \%$ belonged to parity $2 \& 3$. Parity 2 and 3 have been combined as the number of piglets belonging to parity 3 is very small.

\subsection{Least Squares Analysis}

Body Weights

The method of unequal subclass analysis of variance was used to test the significance of the fixed effects. The overall least squares mean body weights $(\mathrm{kgs}) \pm \mathrm{SE}$ at $\operatorname{birth}, 1^{\text {st }}, 2^{\text {nd }}, 3^{\text {rd }}, 4^{\text {th }}, 5^{\text {th }}, 6^{\text {th }}, 7^{\text {th }}, 8^{\text {th }}, 12^{\text {th }}$, $16^{\text {th }}, 20^{\text {th }}, 24^{\text {th }}, 28^{\text {th }}, 32^{\text {nd }}$ and $36^{\text {th }}$ weeks were $1.090 \pm 0.008,1.937 \pm 0.032,2.764 \pm 0.045,3.767 \pm 0.056$, $4.518 \pm 0.077,5.461 \pm 0.077,6.591 \pm 0.070,7.584 \pm 0.126, \quad 8.670 \pm 0.119,12.959 \pm 0.210,18.211 \pm 0.330$, $24.254 \pm 0.478,34.052 \pm 0.617,41.970 \pm 0.741,48.414 \pm 0.941$, and $59.889 \pm 0.828$ respectively (Table 1 and 2). The values were in close agreement with the values reported by $[5,6]$. Genetic group did not affect the preweaning body weights except birth weight and weaning weight. However, significant effect of genetic group was observed on all the post-weaning body weights. Significant effect of genetic group on all body weights except birth weight was reported by [6]. The $75 \% \mathrm{CB}$ recorded heavier body weights at all age groups indicating their superiority over $50 \% \mathrm{CB}$, a finding concurred by authors $[5,7]$ in such similar studies. Even though males were slightly heavier than females during pre-weaning period and up to 5 months of age, females were slightly heavier from the $6^{\text {th }}$ month. However, most of the differences were found to be non-significant. Significant effect of sex on post-weaning weights was reported by [8]. Litter size at birth significantly influenced both the pre and post weaning body weights at all ages. The mean body weight decreased as the litter size at birth increased and possibly due to competition for the resources within the litter. Parity significantly influenced the pre-weaning body weights except at birth and at weaning. The mean body weight of piglets of parity 1 was more than the mean body weight of piglets of parity 2 during pre-weaning period. Similar finding was reported by [7]. As far as post-weaning body weights were concerned, the difference in mean body weights were not significant after 5 months of age. Period of birth had a significant effect on both the pre-weaning and post-weaning mean body weights. The mean body weight increased as the period increased for all ages indicating clearly the constant improvement in management practices over the years yielding good results. Season of birth had a significant effect on the mean body weight of all ages except during first and second week of age. Significant effect of season of birth on the pre-weaning body weights were reported by $[6,9]$.

\section{Average Daily Gain}

The overall least squares mean Average Daily Gains (ADG in kgs.) \pm SE during 0-1, 1-2, 2- 3, 3-4, 4-5, $5-6,6-7,7-8,0-8,8-12,12-16,16-20,20-24,24-28,28-32,32-36,8-36$ weeks were $0.123 \pm 0.004,0.118 \pm$ $0.004,0.117 \pm 0.005,0.137 \pm 0.006,0.138 \pm 0.006,0.152 \pm 0.006,0.155 \pm 0.007,0.191 \pm 0.10,0.135 \pm 0.002$, $0.119 \pm 0.005,0.164 \pm 0.006,0.180 \pm 0.007,0.318 \pm 0.010,0.249 \pm 0.009,0.201 \pm 0.012,0.272 \pm 0.010$ and $0.255 \pm 0.005$ respectively (Table 3 and 4 ) with the maximum average daily gain of 318 gms during $5^{\text {th }}$ to $6^{\text {th }}$ month of age. The effect of genetic group was not significant during pre-weaning periods except during $0-1$ and 0-8 weeks while the genetic group had a significant effect during most of the post-weaning periods. The $75 \%$ $\mathrm{CB}$ recorded faster growth of $140 \mathrm{gms} /$ day and $282 \mathrm{gms} /$ day during pre-weaning $(0-8$ weeks) and post weaning (8-36 weeks) periods as against $130 \mathrm{gms} /$ day and $229 \mathrm{gms} / \mathrm{day}$ for $50 \% \mathrm{CB}$ during the corresponding periods. There was no significant difference in ADG observed between the two sexes during the pre-weaning period and some of the post-weaning periods. However, there was no clear association between gender and ADG. Parity significantly influenced ADG for most of the pre and post-weaning periods except 6-7 and 0-8. The ADG during birth to weaning was 136 gms for parity 1 and 135 gms for parity 2+. Similarly, the ADG during 8-36 weeks was 267 gms for parity 1 and 244 gms for parity 2. Season and Period of birth had a significant effect on the ADG during most of the age periods studied.

\subsection{Heritability, Genotypic and Phenotypic correlations}

The mixed full-sib model considering genetic group, sex, parity, season of birth, litter size at birth as fixed effects were used to estimate the heritability \pm SE for body weights recorded at birth, $1^{\text {st }}, 2^{\text {nd }}, 3^{\text {rd }}, 4^{\text {th }}, 5^{\text {th }}$, $6^{\text {th }}, 7^{\text {th }}, 8^{\text {th }}, 12^{\text {th }}, 16^{\text {th }}, 20^{\text {th }}, 24^{\text {th }}, 28^{\text {th }}, 32^{\text {nd }}$ and $36^{\text {th }}$ week. The results obtained are represented in the diagonal elements of Table 5 . The heritability estimates using sire and dam component for the body weights at birth, $1^{\text {st }}$, 
Factors influencing growth performance and estimation of genetic parameters in crossbred pigs

$2^{\text {nd }}, 3^{\text {rd }}, 4^{\text {th }}, 5^{\text {th }}, 6^{\text {th }}, 7^{\text {th }}, 8^{\text {th }}, 12^{\text {th }}, 16^{\text {th }}, 20^{\text {th }}, 24^{\text {th }}, 28^{\text {th }}$ and $36^{\text {th }}$ week were $0.718,0.834,0.683,0.691,0.577$, $0.597,0.647,0.632,0.880,0.851,0.966,0.907,0.889$ and 0.854 . The estimate obtained for $32^{\text {nd }}$ week was outside the range. The estimates of heritability indicate that post-weaning body weights are more heritable than pre-weaning body weights. Selection for body weights may be very effective from $3^{\text {rd }}$ month.

The estimates of genetic and phenotypic correlation between the bodyweights recorded at various ages obtained through full-sib mixed model are given in upper and lower diagonals respectively of Table 5. The general trend is that as the time gap increases, both the genetic and phenotypic correlation decreases. Estimated genetic correlations among pre-weaning bodyweights were positive and low in magnitude while among postweaning body weights, genetic correlations were high positive. The genetic correlation between birth weight and $3^{\text {rd }}$ month is 0.422 and between $3^{\text {rd }}$ month and $36^{\text {th }}$ month is 0.553 . The finding suggests that birth weight together with $12^{\text {th }}$ week body weight can be used for selection to bring about increased body weight at $36^{\text {th }}$ week.

\section{Tables}

Table 1: Least-squares mean \pm standard error of pre-weaning body weight $(\mathrm{kg})$ of crossbred pigs

(Number of observations in parentheses)

\begin{tabular}{|c|c|c|c|c|c|}
\hline Fixed Effect & Birth Weight (1405) & BW1 (967) & BW2 (965) & BW3 (1071) & BW4 (954) \\
\hline Overall & $1.091 \pm .012$ & $1.979 \pm .034$ & $2.827 \pm .046$ & $3.845 \pm .059$ & $4.628 \pm .073$ \\
\hline Genetic Group & ** & NS & NS & NS & NS \\
\hline $50 \% \mathrm{CB}$ & $1.028 \pm .015^{\mathrm{a}}(388)$ & $1.986 \pm .039(357)$ & $2.805 \pm .053(356)$ & $3.804 \pm .070(357)$ & $4.591 \pm .084(357)$ \\
\hline $75 \% \mathrm{CB}$ & $1.154 \pm .013^{\mathrm{b}}(1022)$ & $1.972 \pm .037(610)$ & $2.849 \pm .051(609)$ & $3.886 \pm .066(714)$ & $4.666 \pm .081(597)$ \\
\hline Sex of Piglet & $*$ & NS & NS & NS & NS \\
\hline Male & $1.106 \pm .013^{\mathrm{a}}(768)$ & $2.003 \pm .036(498)$ & $2.847 \pm .050(496)$ & $3.836 \pm .066(540)$ & $4.662 \pm .080(485)$ \\
\hline Female & $1.076 \pm .013^{\mathrm{b}}(637)$ & $1.955 \pm .038(469)$ & $2.807 \pm .052(469)$ & $3.854 \pm .065(531)$ & $4.595 \pm .083(469)$ \\
\hline Litter Size at Birth & NS & NS & ** & ** & ** \\
\hline $1-3$ & $1.121 \pm .027^{\mathrm{a}}(70)$ & $2.077 \pm .068(66)$ & $3.092 \pm .093^{\mathrm{a}}(64)$ & $4.360 \pm .129^{\mathrm{a}}(63)$ & $5.449 \pm .149^{\mathrm{a}}(63)$ \\
\hline $4-6$ & $1.102 \pm .015^{\mathrm{a}}(350)$ & $1.981 \pm .041(259)$ & $2.868 \pm .056^{\mathrm{a}}(259)$ & $3.929 \pm .073^{\mathrm{b}}(279)$ & $4.825 \pm .088^{\mathrm{b}}(257)$ \\
\hline $7-9$ & $1.084 \pm .013^{\mathrm{a}}(669)$ & $1.981 \pm .034(508)$ & $2.757 \pm .047^{\mathrm{ba}}(508)$ & $3.667 \pm .061^{\mathrm{c}}(551)$ & $4.342 \pm .074^{\circ}(501)$ \\
\hline $10+$ & $1.057 \pm .016^{\mathrm{a}}(316)$ & $1.878 \pm .054(134)$ & $2.592 \pm .073^{\mathrm{b}}(134)$ & $3.422 \pm .089^{\mathrm{d}}(178)$ & $3.898 \pm .115^{\mathrm{d}}(133)$ \\
\hline Parity & $*$ & ** & ** & $* *$ & ** \\
\hline 1 & $1.095 \pm .012(902)$ & $2.080 \pm .039^{\mathrm{a}}(636)$ & $3.042 \pm .053^{\mathrm{a}}(635)$ & $4.270 \pm .062^{\mathrm{a}}(745)$ & $5.106 \pm .085^{\mathrm{a}}(626)$ \\
\hline $2+$ & $1.087 \pm .014(503)$ & $1.878 \pm .041^{\mathrm{b}}(331)$ & $2.612 \pm .056^{\mathrm{b}}(330)$ & $3.419 \pm .078^{\mathrm{b}}(326)$ & $4.150 \pm .089^{b}(328)$ \\
\hline Season of Birth & ** & NS & NS & $* *$ & NS \\
\hline Summer(3-5) & $1.086 \pm .014^{\mathrm{a}}(484)$ & $1.889 \pm .053(397)$ & $2.770 \pm .072(397)$ & $3.266 \pm .071^{\mathrm{a}}(392)$ & $4.429 \pm .113(394)$ \\
\hline Pre-Monsoon(6-8) & $1.062 \pm .017^{\mathrm{a}}(277)$ & $2.004 \pm .063(142)$ & $2.840 \pm .086(142)$ & $4.070 \pm .101^{\mathrm{b}}(142)$ & $4.659 \pm .135(142)$ \\
\hline Monsoon (9-11) & $1.158 \pm .012^{\mathrm{b}}(602)$ & $1.979 \pm .045(386)$ & $2.790 \pm .061(385)$ & $4.022 \pm .059^{\mathrm{b}}(502)$ & $4.563 \pm .096(380)$ \\
\hline Winter(12-2) & $1.058 \pm .036^{\mathrm{a}}(42)$ & $2.044 \pm .089(42)$ & $2.908 \pm .122(41)$ & $4.020 \pm .172^{\mathrm{b}}(35)$ & $4.863 \pm .198(38)$ \\
\hline Period of Birth & NS & $* *$ & $* *$ & $* *$ & $* *$ \\
\hline $2002-2005$ & $1.081 \pm .013^{\mathrm{a}}(654)$ & $1.823 \pm .042(528)$ & $2.600 \pm .057^{\mathrm{a}}(526)$ & $3.087 \pm .064^{\mathrm{a}}(515)$ & $4.035 \pm .090^{\mathrm{a}}(518)$ \\
\hline $2006-2011$ & $1.101 \pm .016^{\mathrm{b}}(751)$ & $2.136 \pm .070(439)$ & $3.054 \pm .094^{\mathrm{b}}(439)$ & $4.602 \pm .084^{\mathrm{b}}(556)$ & $5.222 \pm .149^{\mathrm{b}}(436)$ \\
\hline
\end{tabular}

Table 1 (cont.): Least-squares mean \pm standard error of pre-weaning body weight $(\mathrm{kg})$ of crossbred pigs (Number of observations in parentheses)

\begin{tabular}{|l|l|l|l|l|}
\hline Fixed Effect & BW5 $(1089)$ & BW6 $(1099)$ & BW7 $(1055)$ & BW8 (1405) \\
\hline Overall & $5.641 \pm .077$ & $6.598 \pm .087$ & $7.823 \pm .105$ & $9.264 \pm .119$ \\
\hline Genetic Group & NS & $* *$ & NS & $* *$ \\
\hline $50 \%$ CB & $5.581 \pm .094(357)$ & $6.567 \pm .106^{\mathrm{a}}(357)$ & $7.750 \pm .128(357)$ & $9.046 \pm .150^{\mathrm{a}}(383)$ \\
\hline $75 \%$ CB & $5.702 \pm .085(732)$ & $6.628 \pm .098^{\mathrm{b}}(742)$ & $7.896 \pm .116(698)$ & $9.483 \pm .130^{\mathrm{b}}(1022)$ \\
\hline Sex of Piglet & NS & NS & NS & $*$ \\
\hline Male & $5.648 \pm .087(559)$ & $6.618 \pm .097(604)$ & $7.858 \pm .118(550)$ & $9.385 \pm .133^{\mathrm{a}}(768)$ \\
\hline Female & $5.635 \pm .087(530)$ & $6.577 \pm .101(495)$ & $7.789 \pm .120(505)$ & $9.143 \pm .136^{\mathrm{b}}(637)$ \\
\hline Litter Size at Birth & $* *$ & $* *$ & $* *$ \\
\hline $1-3$ & $6.845 \pm .171^{\mathrm{a}}(66)$ & $7.907 \pm .195^{\mathrm{a}}(66)$ & $9.195 \pm .233^{\mathrm{a}}(66)$ & $10.509 \pm .281^{\mathrm{a}}(70)$ \\
\hline $4-6$ & $5.863 \pm .098^{\mathrm{b}}(280)$ & $6.908 \pm .110^{\mathrm{b}}(291)$ & $8.002 \pm .136^{\mathrm{b}}(268)$ & $9.521 \pm .152^{\mathrm{b}}(350)$ \\
\hline $7-9$ & $5.247 \pm .081^{\mathrm{c}}(564)$ & $6.207 \pm .093^{\mathrm{c}}(557)$ & $7.408 \pm .113^{\mathrm{c}}(530)$ & $8.805 \pm .130^{\mathrm{c}}(669)$ \\
\hline $10+$ & $4.611 \pm .122^{\mathrm{d}}(179)$ & $5.368 \pm .139^{\mathrm{d}}(185)$ & $6.688 \pm .162^{\mathrm{d}}(191)$ & $8.222 \pm .167^{\mathrm{d}}(316)$ \\
\hline Parity & $* *$ & $*$ & $\mathrm{NS}$ \\
\hline 1 & $6.243 \pm .081^{\mathrm{a}}(759)$ & $7.279 \pm .091^{\mathrm{a}}(769)$ & $8.413 \pm .114^{\mathrm{a}}(702)$ & $9.381 \pm .129(902)$ \\
\hline $2+$ & $5.040 \pm .104^{\mathrm{b}}(330)$ & $5.916 \pm .117^{\mathrm{b}}(330)$ & $7.233 \pm .138^{\mathrm{b}}(353)$ & $9.147 \pm .148(503)$ \\
\hline Season of Birth & $* *$ & $\mathrm{NS}$ & $*$ & $* *$ \\
\hline Summer(3-5) & $5.272 \pm .097^{\mathrm{a}}(396)$ & $6.331 \pm .111(446)$ & $7.237 \pm .140^{\mathrm{a}}(419)$ & $8.597 \pm .146^{\mathrm{a}}(484)$ \\
\hline Pre-Monsoon(6-8) & $5.640 \pm .138^{\mathrm{ab}}(142)$ & $6.487 \pm .144(162)$ & $8.064 \pm .192^{\mathrm{b}}(142)$ & $9.523 \pm .177^{\mathrm{b}}(277)$ \\
\hline
\end{tabular}


Factors influencing growth performance and estimation of genetic parameters in crossbred pigs

\begin{tabular}{|l|l|l|l|l|}
\hline Monsoon (9-11) & $5.627 \pm .080^{\mathrm{b}}(509)$ & $6.543 \pm .099(449)$ & $7.817 \pm .116^{\mathrm{b}}(452)$ & $9.500 \pm .122^{\mathrm{b}}(602)$ \\
\hline Winter(12-2) & $6.026 \pm .217^{\mathrm{b}}(42)$ & $7.030 \pm .249(42)$ & $8.174 \pm .297^{\mathrm{b}}(42)$ & $9.437 \pm .369^{\mathrm{ab}}(42)$ \\
\hline Period of Birth & $* *$ & $* *$ & $* *$ & $* *$ \\
\hline $2002-2005$ & $4.826 \pm .088^{\mathrm{a}}(528)$ & $5.686 \pm .103^{\mathrm{a}}(528)$ & $6.637 \pm .123^{\mathrm{a}}(528)$ & $8.367 \pm .133^{\mathrm{a}}(654)$ \\
\hline $2006-2011$ & $6.457 \pm .113^{\mathrm{b}}(561)$ & $7.509 \pm .132^{\mathrm{b}}(571)$ & $9.009 \pm .169^{\mathrm{b}}(527)$ & $10.162 \pm .166^{\mathrm{b}}(751)$ \\
\hline
\end{tabular}

NS - not significant; * significant $(\mathrm{P}<0.05)$; ** significant $(\mathrm{P}<0.01)$
Means with different superscripts under each effect within the column differ significantly.

Table 2: Least-squares mean \pm standard error of post-weaning body weight $(\mathrm{kg})$ of crossbred pigs

(Number of observations in parentheses)

\begin{tabular}{|l|l|l|l|l|}
\hline Fixed Effect & BW12 $(981)$ & BW16 $(874)$ & BW20 $(773)$ & BW24 $(716)$ \\
\hline Overall & $13.244 \pm .214$ & $18.276 \pm .352$ & $24.256 \pm 0.509$ & $34.458 \pm 0.660$ \\
\hline Genetic Group & $* *$ & $* *$ & $* *$ & $* *$ \\
\hline $50 \%$ CB & $12.763 \pm .273^{\mathrm{a}}(260)$ & $17.427 \pm .454^{\mathrm{a}}(246)$ & $22.501 \pm 0.659^{\mathrm{a}}(224)$ & $32.531 \pm 0.860^{\mathrm{a}}(200)$ \\
\hline $75 \%$ CB & $13.725 \pm .240^{\mathrm{b}}(721)$ & $19.125 \pm .394^{\mathrm{b}}(628)$ & $26.011 \pm 0.568^{\mathrm{b}}(549)$ & $36.386 \pm 0.746^{\mathrm{b}}(516)$ \\
\hline Sex of Piglet & NS & NS & $*$ & NS \\
\hline Male & $13.246 \pm .236(536)$ & $18.461 \pm .392(461)$ & $24.917 \pm 0.575(392)$ & $34.070 \pm 0.754(353)$ \\
\hline Female & $13.242 \pm .247(445)$ & $18.090 \pm .411(413)$ & $23.595 \pm 0.592(381)$ & $34.847 \pm 0.770(363)$ \\
\hline Litter Size at Birth & $* *$ & NS & NS & NS \\
\hline $1-3$ & $13.831 \pm .536^{\mathrm{a}}(48)$ & $18.399 \pm 0.888(45)$ & $25.605 \pm 1.309(40)$ & $36.741 \pm 1.758(36)$ \\
\hline $4-6$ & $13.683 \pm .28^{\mathrm{ab}}(244)$ & $18.290 \pm 0.467(222)$ & $23.954 \pm 0.665(207)$ & $33.031 \pm 0.885(186)$ \\
\hline $7-9$ & $12.816 \pm .24^{\mathrm{ac}}(459)$ & $17.821 \pm .398(404)$ & $23.114 .573(360)$ & $32.839 \pm 0.745(343)$ \\
\hline $10+$ & $12.645 \pm .29^{\mathrm{ac}}(230)$ & $18.593 \pm .497(203)$ & $24.351 \pm .737(166)$ & $35.222 \pm 0.975(151)$ \\
\hline Parity & $* *$ & $* *$ & $* *$ & NS \\
\hline 1 & $12.754 \pm .221^{\mathrm{a}}(704)$ & $17.062 \pm .369^{\mathrm{a}}(612)$ & $22.744 \pm .558^{\mathrm{a}}(520)$ & $34.270 \pm 0.738(464)$ \\
\hline $2+$ & $13.734 \pm .282^{\mathrm{b}}(277)$ & $19.489 \pm .466^{\mathrm{b}}(262)$ & $25.768 \pm .661^{\mathrm{b}}(253)$ & $34.647 \pm 0.852(252)$ \\
\hline Season of Birth & $* *$ & $* *$ & $* *$ \\
\hline Summer(3-5) & $12.473 \pm .32^{\mathrm{ab}}(194)$ & $17.005 \pm .529^{\mathrm{a}}(187)$ & $24.129 \pm 0.789^{\mathrm{a}}(156)$ & $37.595 \pm 1.086^{\mathrm{a}}(128)$ \\
\hline Pre-Monsoon(6-8) & $13.678 \pm .298^{\mathrm{b}}(273)$ & $19.630 \pm .519^{\mathrm{b}}(230)$ & $25.454 \pm 0.811^{\mathrm{a}}(195)$ & $35.278 \pm 1.066^{\mathrm{a}}(194)$ \\
\hline Monsoon $(9-11)$ & $14.257 \pm .209^{\mathrm{b}}(476)$ & $19.789 \pm .351^{\mathrm{b}}(420)$ & $27.177 \pm 0.511^{\mathrm{ba}}(387)$ & $37.241 \pm 0.687^{\mathrm{a}}(358)$ \\
\hline Winter(12-2) & $12.568 \pm .605^{\mathrm{ab}}(38)$ & $16.68 \pm 0.990^{\mathrm{a}}(37)$ & $20.265 \pm 1.413^{\mathrm{a}}(35)$ & $27.720 \pm 1.802^{\mathrm{b}}(36)$ \\
\hline Period of Birth & $* *$ & $* *$ & $* *$ & $* *$ \\
\hline $2002-2005$ & $11.540 \pm .230^{\mathrm{a}}(547)$ & $15.918 \pm .382^{\mathrm{a}}(494)$ & $21.724 \pm 0.557^{\mathrm{a}}(456)$ & $31.836 \pm 0.733^{\mathrm{a}}(429)$ \\
\hline $2006-2011$ & $14.948 \pm .300^{\mathrm{b}}(434)$ & $20.634 \pm .507^{\mathrm{b}}(380)$ & $26.788 \pm 0.750^{\mathrm{b}}(317)$ & $37.081 \pm 0.979^{\mathrm{b}}(287)$ \\
\hline \multicolumn{5}{|l|}{ NS - not $^{\mathrm{b}}$ significant $; *$ significant $(\mathrm{P}<0.05) ; * *$ significant $(\mathrm{P}<0.01)}$. \\
\\
\hline
\end{tabular}

Table 2 (contd.): Least-squares mean \pm standard error of post-weaning body weight $(\mathrm{kg})$ of crossbred pigs (Number of observations in parentheses)

\begin{tabular}{|c|c|c|c|}
\hline Fixed Effect & BW28 (692) & BW32 (603) & BW36 (445) \\
\hline Overall & $42.411 \pm 0.789$ & $48.491 \pm 1.018$ & $59.936 \pm 0.983$ \\
\hline Genetic Group & $* *$ & $* *$ & $* *$ \\
\hline $50 \% \mathrm{CB}$ & $40.262 \pm 1.030^{\mathrm{a}}(192)$ & $45.019 \pm 1.319^{\mathrm{a}}(164)$ & $53.912 \pm 1.352^{\mathrm{a}}(123)$ \\
\hline $75 \% \mathrm{CB}$ & $44.560 \pm 0.877^{\mathrm{b}}(500)$ & $51.964 \pm 1.107^{\mathrm{b}}(439)$ & $65.959 \pm 1.100^{\mathrm{b}}(322)$ \\
\hline Sex of Piglet & $* *$ & NS & NS \\
\hline Male & $41.006 \pm 0.904^{\mathrm{a}}(339)$ & $47.637 \pm 1.183^{\mathrm{a}}(290)$ & $59.419 \pm 1.224(220)$ \\
\hline Female & $43.816 \pm 0.915^{\mathrm{b}}(353)$ & $49.346 \pm 1.152^{b}(313)$ & $60.452 \pm 1.138(225)$ \\
\hline Litter Size at Birth & $* *$ & $*$ & NS \\
\hline $1-3$ & $45.481 \pm 2.084^{\mathrm{a}}(34)$ & $51.620 \pm 2.691^{\mathrm{a}}(28)$ & $63.923 \pm 3.254(17)$ \\
\hline $4-6$ & $39.939 \pm 1.038^{\mathrm{a}}(184)$ & $45.681 \pm 1.293^{\mathrm{ab}}(167)$ & $57.829 \pm 1.259(114)$ \\
\hline $7-9$ & $40.172 \pm 0.895^{\mathrm{a}}(326)$ & $46.164 \pm 1.143^{\mathrm{a}}(282)$ & $58.117 \pm 1.003(210)$ \\
\hline $10+$ & $44.051 \pm 1.143^{\mathrm{ab}}(148)$ & $50.501 \pm 1.441^{\mathrm{ac}}(126)$ & $59.874 \pm 1.454(104)$ \\
\hline Parity & NS & NS & $*$ \\
\hline 1 & $43.179 \pm 0.882^{\mathrm{a}}(446)$ & $49.469 \pm 1.135(373)$ & $61.789 \pm 1.252^{\mathrm{a}}(241)$ \\
\hline $2+$ & $41.643 \pm 1.011^{\mathrm{b}}(246)$ & $47.513 \pm 1.283(230)$ & $58.082 \pm 1.194^{\mathrm{b}}(204)$ \\
\hline Season of Birth & $* *$ & $* *$ & $* *$ \\
\hline Summer(3-5) & $46.554 \pm 1.269^{\mathrm{a}}(127)$ & $49.571 \pm 1.573^{\mathrm{a}}(120)$ & $57.247 \pm 1.720^{\mathrm{a}}(98)$ \\
\hline Pre-Monsoon(6-8) & $47.700 \pm 1.247^{\mathrm{a}}(194)$ & $54.418 \pm 1.524^{\mathrm{a}}(188)$ & $58.842 \pm 1.733^{\mathrm{a}}(161)$ \\
\hline Monsoon (9-11) & $45.440 \pm 0.815^{\mathrm{a}}(338)$ & $53.918 \pm 1.058^{\mathrm{a}}(267)$ & $63.718 \pm 1.172^{b}(186)$ \\
\hline Winter(12-2) & $31.950 \pm 2.171^{\mathrm{b}}(33)$ & $36.058 \pm 2.786^{\mathrm{b}}(28)$ & - \\
\hline Period of Birth & $* *$ & $* *$ & $* *$ \\
\hline $2002-2005$ & $38.480 \pm 0.869^{\mathrm{a}}(420)$ & $43.573 \pm 1.117^{\mathrm{a}}(380)$ & $56.676 \pm 1.286^{\mathrm{a}}(261)$ \\
\hline $2006-2011$ & $46.342 \pm 1.174^{\mathrm{b}}(272)$ & $53.409 \pm 1.523^{\mathrm{b}}(223)$ & $63.195 \pm 1.479^{b}(184)$ \\
\hline
\end{tabular}


Factors influencing growth performance and estimation of genetic parameters in crossbred pigs

Table 3: Least-squares mean \pm standard error of pre-weaning Average Daily Gains (kgs.) of crossbred pigs

(Number of observations in parentheses)

\begin{tabular}{|l|l|l|l|l|}
\hline Fixed Effect & ADG01 $(967)$ & ADG12 $(965)$ & ADG23 $(948)$ & ADG34 (946) \\
\hline Overall & $0.128 \pm .004$ & $0.121 \pm .004$ & $0.122 \pm .005$ & $0.139 \pm .005$ \\
\hline Genetic Group & $* *$ & N & NS & NS \\
\hline $50 \%$ CB & $0.137 \pm .005^{\mathrm{a}}(357)$ & $0.117 \pm .005(356)$ & $0.121 \pm .006(356)$ & $0.137 \pm .006(357)$ \\
\hline $75 \%$ CB & $0.119 \pm .005^{\mathrm{b}}(610)$ & $0.125 \pm .005(609)$ & $0.124 \pm .005(592)$ & $0.141 \pm .006(589)$ \\
\hline Sex of Piglet & $\mathrm{NS}$ & $\mathrm{NS}$ & $\mathrm{NS}$ & $\mathrm{NS}$ \\
\hline Male & $0.130 \pm .005(498)$ & $0.121 \pm .005(496)$ & $0.122 \pm .005(479)$ & $0.143 \pm .006(477)$ \\
\hline Female & $0.126 \pm .005(469)$ & $0.122 \pm .005(469)$ & $0.123 \pm .005(469)$ & $0.135 \pm .006(469)$ \\
\hline Litter Size at Birth & $\mathrm{NS}$ & $* *$ & $* *$ & $* *$ \\
\hline $1-3$ & $0.137 \pm .008(66)$ & $0.145 \pm .009^{\mathrm{a}}(64)$ & $0.165 \pm .010^{\mathrm{a}}(62)$ & $0.181 \pm .010^{\mathrm{a}}(63)$ \\
\hline $4-6$ & $0.126 \pm .005(259)$ & $0.127 \pm .005^{\mathrm{a}}(259)$ & $0.130 \pm .006^{\mathrm{b}}(257)$ & $0.152 \pm .006^{\mathrm{b}}(257)$ \\
\hline $7-9$ & $0.129 \pm .004(508)$ & $0.111 \pm .004^{\mathrm{b}}(508)$ & $0.113 \pm .005^{\mathrm{c}}(496)$ & $0.117 \pm .005^{\mathrm{c}}(493)$ \\
\hline $10+$ & $0.120 \pm .007(134)$ & $0.102 \pm .007^{\mathrm{b}}(134)$ & $0.082 \pm .008^{\mathrm{d}}(133)$ & $0.105 \pm .008^{\mathrm{c}}(133)$ \\
\hline Parity & $* *$ & $* *$ & $* *$ & $* *$ \\
\hline 1 & $0.140 \pm .005^{\mathrm{a}}(636)$ & $0.138 \pm .005^{\mathrm{a}}(635)$ & $0.140 \pm .006^{\mathrm{a}}(623)$ & $0.158 \pm .006^{\mathrm{a}}(620)$ \\
\hline $2+$ & $0.117 \pm .005^{\mathrm{b}}(331)$ & $0.105 \pm .005^{\mathrm{b}}(330)$ & $0.105 \pm .006^{\mathrm{b}}(325)$ & $0.120 \pm .006^{\mathrm{b}}(326)$ \\
\hline Season of Birth & $\mathrm{NS}$ & $\mathrm{NS}$ & $\mathrm{NS}$ & $* *$ \\
\hline Summer(3-5) & $0.115 \pm .007(397)$ & $0.126 \pm .007(397)$ & $0.127 \pm .007(392)$ & $0.113 \pm .008^{\mathrm{a}}(392)$ \\
\hline Pre-Monsoon(6-8) & $0.135 \pm .008(142)$ & $0.119 \pm .008(142)$ & $0.125 \pm .009(142)$ & $0.135 \pm .009^{\mathrm{a}}(142)$ \\
\hline Monsoon $(9-11)$ & $0.120 \pm .006(386)$ & $0.116 \pm .006(385)$ & $0.127 \pm .006(379)$ & $0.126 \pm .007^{\mathrm{a}}(377)$ \\
\hline Winter(12-2) & $0.143 \pm .011(42)$ & $0.124 \pm .012(41)$ & $0.112 \pm .013(35)$ & $0.182 \pm .014^{\mathrm{b}}(35)$ \\
\hline Period of Birth & $* *$ & $\mathrm{NS}$ & $* *$ & $* *$ \\
\hline $2002-2005$ & $0.107 \pm .005^{\mathrm{a}}(528)$ & $0.111 \pm .005(526)$ & $0.103 \pm .006^{\mathrm{a}}(514)$ & $0.107 \pm .006^{\mathrm{a}}(512)$ \\
\hline $2006-2011$ & $0.149 \pm .009^{\mathrm{b}}(439)$ & $0.131 \pm .009(439)$ & $0.141 \pm .010^{\mathrm{b}}(434)$ & $0.171 \pm .010^{\mathrm{b}}(434)$ \\
\hline
\end{tabular}

NS - not significant ; * significant $(\mathrm{P}<0.05) ; * *$ significant $(\mathrm{P}<0.01)$

Means with different superscripts under each effect within the column differ significantly.

Table 3 (contd.): Least-squares mean \pm standard error of pre-weaning Average Daily Gains (kg) of crossbred pigs (Number of observations in parentheses)

\begin{tabular}{|c|c|c|c|c|c|}
\hline Fixed Effect & ADG45 (953) & ADG56 (1023) & ADG67 (966) & ADG78 (1055) & ADG08 (1405) \\
\hline Overall & $0.152 \pm .006$ & $0.156 \pm .006$ & $0.162 \pm .007$ & $0.204 \pm .010$ & $0.146 \pm .002$ \\
\hline Genetic Group & NS & $*$ & NS & NS & * \\
\hline $50 \% \mathrm{CB}$ & $0.147 \pm .007(357)$ & $0.163 \pm .007^{\mathrm{a}}(357)$ & $0.157 \pm .008(357)$ & $0.199 \pm .012(357)$ & $0.143 \pm .003^{\mathrm{a}}(383)$ \\
\hline $75 \% \mathrm{CB}$ & $0.157 \pm .006(596)$ & $0.149 \pm .007^{\mathrm{b}}(666)$ & $0.167 \pm .008(609)$ & $0.209 \pm .011(698)$ & $0.149 \pm .002^{\mathrm{b}}(1022)$ \\
\hline Sex of Piglet & NS & NS & NS & NS & NS \\
\hline Male & $0.150 \pm .006(485)$ & $0.154 \pm .007(528)$ & $0.158 \pm .008(498)$ & $0.211 \pm .011(550)$ & $0.148 \pm .002(768)$ \\
\hline Female & $0.155 \pm .006(468)$ & $0.158 \pm .007(495)$ & $0.166 \pm .008(468)$ & $0.197 \pm .011(505)$ & $0.144 \pm .002(637)$ \\
\hline Litter Size at Birth & $* *$ & NS & $* *$ & NS & $* *$ \\
\hline $1-3$ & $0.213 \pm .012^{\mathrm{a}}(63)$ & $0.171 \pm .013(66)$ & $0.179 \pm .014^{\mathrm{a}}(66)$ & $0.197 \pm .022(66)$ & $0.168 \pm .005^{\mathrm{a}}(70)$ \\
\hline $4-6$ & $0.158 \pm .007^{b}(256)$ & $0.151 \pm .008(270)$ & $0.178 \pm .009^{\mathrm{a}}(258)$ & $0.209 \pm .013(268)$ & $0.150 \pm .003^{\mathrm{b}}(350)$ \\
\hline $7-9$ & $0.141 \pm .006^{b}(501)$ & $0.158 \pm .006(543)$ & $0.156 \pm .007^{\mathrm{ab}}(508)$ & $0.204 \pm .011(530)$ & $0.138 \pm .002^{\mathrm{c}}(669)$ \\
\hline $10+$ & $0.096 \pm .009^{\mathrm{c}}(133)$ & $0.143 \pm .010(144)$ & $0.136 \pm .011^{\mathrm{ab}}(134)$ & $0.204 \pm .015(191)$ & $0.128 \pm .003^{\mathrm{d}}(316)$ \\
\hline Parity & $* *$ & $* *$ & NS & $*$ & $\mathrm{NS}$ \\
\hline 1 & $0.175 \pm .007^{\mathrm{a}}(626)$ & $0.168 \pm .007^{\mathrm{a}}(693)$ & $0.168 \pm .008(636)$ & $0.188 \pm .011^{\mathrm{a}}(702)$ & $0.148 \pm .002(902)$ \\
\hline $2+$ & $0.129 \pm .007^{b}(327)$ & $0.144 \pm .008^{\mathrm{b}}(330)$ & $0.156 \pm .009(330)$ & $0.219 \pm .013^{\mathrm{b}}(353)$ & $0.144 \pm .003(503)$ \\
\hline Season of Birth & $* *$ & $* *$ & $* *$ & $* *$ & $* *$ \\
\hline Summer(3-5) & $0.101 \pm .009^{\mathrm{a}}(393)$ & $0.111 \pm .008^{\mathrm{a}}(396)$ & $0.116 \pm .011^{\mathrm{a}}(396)$ & $0.113 \pm .013^{\mathrm{a}}(419)$ & $0.134 \pm .003^{\mathrm{a}}(484)$ \\
\hline Pre-Monsoon(6-8) & $0.152 \pm .011^{\mathrm{b}}(142)$ & $0.182 \pm .011^{\mathrm{b}}(142)$ & $0.192 \pm .013^{\mathrm{b}}(142)$ & $0.215 \pm .018^{\mathrm{b}}(142)$ & $0.151 \pm .003^{\mathrm{b}}(277)$ \\
\hline Monsoon (9-11) & $0.163 \pm .008^{\mathrm{b}}(380)$ & $0.156 \pm .007^{\mathrm{b}}(443)$ & $0.183 \pm .010^{\mathrm{b}}(386)$ & $0.269 \pm .011^{\mathrm{cb}}(452)$ & $0.149 \pm .002^{\mathrm{b}}(602)$ \\
\hline Winter(12-2) & $0.191 \pm .016^{\mathrm{b}}(38)$ & $0.175 \pm .017^{\mathrm{b}}(42)$ & $0.156 \pm .019^{\mathrm{ab}}(42)$ & $0.218 \pm .028^{\mathrm{c}}(42)$ & $0.150 \pm .006^{\mathrm{ab}}(42)$ \\
\hline Period of Birth & $* *$ & $* *$ & $* *$ & $* *$ & $* *$ \\
\hline $2002-2005$ & $0.101 \pm .007^{\mathrm{a}}(518)$ & $0.110 \pm .007^{\mathrm{a}}(528)$ & $0.112 \pm .009^{\mathrm{a}}(528)$ & $0.157 \pm .012^{\mathrm{a}}(528)$ & $0.130 \pm .002^{\mathrm{a}}(654)$ \\
\hline $2006-2011$ & $0.203 \pm .012^{\mathrm{b}}(435)$ & $0.202 \pm .010^{\mathrm{b}}(495)$ & $0.211 \pm .015^{\mathrm{b}}(438)$ & $0.250 \pm .016^{\mathrm{b}}(527)$ & $0.162 \pm .003^{\mathrm{b}}(751)$ \\
\hline
\end{tabular}

Table 4: Least-squares mean \pm standard error of post-weaning Average Daily Gains $(\mathrm{kg})$ of crossbred pigs (Number of observations in parentheses)

\begin{tabular}{|l|l|l|l|l|}
\hline Fixed Effect & ADG812 (981) & ADG1216 (871) & ADG1620 (762) & ADG2024 (705) \\
\hline Overall & $0.120 \pm .005$ & $0.163 \pm .007$ & $0.179 \pm .008$ & $0.318 \pm .010$ \\
\hline Genetic Group & NS & $* *$ & $* *$ & NS \\
\hline $50 \%$ CB & $0.114 \pm .006(260)$ & $0.148 \pm .009^{\mathrm{a}}(246)$ & $0.145 \pm .010^{\mathrm{a}}(221)$ & $0.308 \pm .014(197)$ \\
\hline $75 \%$ CB & $0.126 \pm .006(721)$ & $0.178 \pm .007^{\mathrm{b}}(625)$ & $0.213 \pm .009^{\mathrm{b}}(541)$ & $0.327 \pm .012(508)$ \\
\hline
\end{tabular}


Factors influencing growth performance and estimation of genetic parameters in crossbred pigs

\begin{tabular}{|l|l|l|l|l|}
\hline Sex of Piglet & NS & NS & NS & $* *$ \\
\hline Male & $0.115 \pm .005(536)$ & $0.166 \pm .007(458)$ & $0.185 \pm .009(384)$ & $0.271 \pm .012^{\mathrm{a}}(348)$ \\
\hline Female & $0.125 \pm .006(445)$ & $0.160 \pm .008(413)$ & $0.173 \pm .009(378)$ & $0.365 \pm .012^{\mathrm{b}}(357)$ \\
\hline Litter Size at Birth & $* *$ & $* *$ & $* *$ & NS \\
\hline $1-3$ & $0.106 \pm .012^{\mathrm{a}}(48)$ & $0.151 \pm .017^{\mathrm{a}}(45)$ & $0.219 \pm .020^{\mathrm{a}}(40)$ & $0.343 \pm .028(35)$ \\
\hline $4-6$ & $0.121 \pm .006^{\mathrm{a}}(244)$ & $0.154 \pm .009^{\mathrm{ab}}(222)$ & $0.172 \pm .010^{\mathrm{a}}(203)$ & $0.286 \pm .014(185)$ \\
\hline $7-9$ & $0.116 \pm .005^{\mathrm{ab}}(459)$ & $0.162 \pm .007^{\mathrm{a}}(404)$ & $0.159 \pm .009^{\mathrm{ba}}(356)$ & $0.311 \pm .012(338)$ \\
\hline $10+$ & $0.137 \pm .007^{\mathrm{ac}}(230)$ & $0.185 \pm .009^{\mathrm{ac}}(200)$ & $0.166 \pm .011^{\mathrm{a}}(163)$ & $0.330 \pm .015(147)$ \\
\hline Parity & $* *$ & $* *$ & $\mathrm{NS}$ & $* *$ \\
\hline 1 & $0.109 \pm .005^{\mathrm{a}}(704)$ & $0.133 \pm .007^{\mathrm{a}}(609)$ & $0.169 \pm .009(518)$ & $0.345 \pm .012^{\mathrm{a}}(453)$ \\
\hline $2+$ & $0.131 \pm .006^{\mathrm{b}}(277)$ & $0.193 \pm .009^{\mathrm{b}}(262)$ & $0.189 \pm .010(244)$ & $0.291 \pm .013^{\mathrm{b}}(252)$ \\
\hline Season of Birth & $* *$ & $\mathrm{NS}$ & $* *$ & $* *$ \\
\hline Summer(3-5) & $0.092 \pm .007^{\mathrm{b}}(194)$ & $0.158 \pm .010(187)$ & $0.198 \pm .013^{\mathrm{a}}(147)$ & $0.394 \pm .017^{\mathrm{a}}(128)$ \\
\hline Pre-Monsoon(6-8) & $0.145 \pm .007^{\mathrm{a}}(273)$ & $0.180 \pm .010(230)$ & $0.165 \pm .013^{\mathrm{ac}}(195)$ & $0.310 \pm .017^{\mathrm{b}}(194)$ \\
\hline Monsoon $(9-11)$ & $0.149 \pm .005^{\mathrm{a}}(476)$ & $0.178 \pm .007(417)$ & $0.240 \pm .008^{\mathrm{b}}(385)$ & $0.325 \pm .011^{\mathrm{bc}}(351)$ \\
\hline Winter(12-2) & $0.093 \pm .014^{\mathrm{b}}(38)$ & $0.136 \pm .019(37)$ & $0.113 \pm .022^{\mathrm{c}}(35)$ & $0.243 \pm .029^{\mathrm{bd}}(32)$ \\
\hline Period of Birth & $* *$ & $* *$ & $\mathrm{NS}$ & $\mathrm{NS}$ \\
\hline $2002-2005$ & $0.091 \pm .005^{\mathrm{a}}(547)$ & $0.143 \pm .007^{\mathrm{a}}(494)$ & $0.180 \pm .009(456)$ & $0.313 \pm .011(425)$ \\
\hline $2006-2011$ & $0.150 \pm .007^{\mathrm{b}}(434)$ & $0.183 \pm .010^{\mathrm{b}}(377)$ & $0.178 \pm .012(306)$ & $0.322 \pm .015(280)$ \\
\hline
\end{tabular}

NS - not significant ; * significant $(\mathrm{P}<0.05)$;** significant $(\mathrm{P}<0.01)$

Means with different superscripts under each effect within the column differ significantly.

Table 4 (contd.): Least-squares mean \pm standard error of post-weaning Average Daily Gains $(\mathrm{kg})$ of crossbred pigs (Number of observations in parentheses)

\begin{tabular}{|l|l|l|l|l|}
\hline Fixed Effect & ADG2428 $(686)$ & ADG2832 $(601)$ & ADG3236 $(445)$ & ADG836 (445) \\
\hline Overall & $0.250 \pm .010$ & $0.195 \pm .013$ & $0.276 \pm .012$ & $0.255 \pm .005$ \\
\hline Genetic Group & NS & $* *$ & $*$ & $* *$ \\
\hline $50 \%$ CB & $0.243 \pm .013(192)$ & $0.173 \pm .016^{\mathrm{a}}(162)$ & $0.251 \pm .017^{\mathrm{a}}(123)$ & $0.227 \pm .007^{\mathrm{a}}(123)$ \\
\hline $75 \%$ CB & $0.258 \pm .011(494)$ & $0.217 \pm .014^{\mathrm{b}}(439)$ & $0.301 \pm .014^{\mathrm{b}}(322)$ & $0.284 \pm .005^{\mathrm{b}}(322)$ \\
\hline Sex of Piglet & $* *$ & $*$ & $\mathrm{NS}$ & $\mathrm{NS}$ \\
\hline Male & $0.221 \pm .011^{\mathrm{a}}(338)$ & $0.211 \pm .015^{\mathrm{a}}(288)$ & $0.276 \pm .015(220)$ & $0.251 \pm .006(220)$ \\
\hline Female & $0.280 \pm .011^{\mathrm{b}}(348)$ & $0.179 \pm .014^{\mathrm{b}}(313)$ & $0.276 \pm .014(225)$ & $0.259 \pm .006(225)$ \\
\hline $\begin{array}{l}\text { Litter Size at } \\
\text { Birth }\end{array}$ & $\mathrm{NS}$ & $\mathrm{NS}$ & $\mathrm{NS}$ & $\mathrm{NS}$ \\
\hline $1-3$ & $0.282 \pm .026(34)$ & $0.186 \pm .033(28)$ & $0.297 \pm .040(17)$ & $0.269 \pm .016(17)$ \\
\hline $4-6$ & $0.227 \pm .013(184)$ & $0.185 \pm .016(165)$ & $0.275 \pm .016(114)$ & $0.242 \pm .006(114)$ \\
\hline $7-9$ & $0.237 \pm .011(326)$ & $0.188 \pm .014(282)$ & $0.277 \pm .012(210)$ & $0.249 \pm .005(210)$ \\
\hline $10+$ & $0.256 \pm .014(142)$ & $0.223 \pm .018(126)$ & $0.256 \pm .018(104)$ & $0.261 \pm .007(104)$ \\
\hline Parity & $* *$ & $\mathrm{NS}$ & $* *$ & $* *$ \\
\hline 1 & $0.273 \pm .011(440)$ & $0.210 \pm .014(373)$ & $0.302 \pm .016^{\mathrm{a}}(241)$ & $0.266 \pm .006^{\mathrm{a}}(241)$ \\
\hline $2+$ & $0.228 \pm .012(246)$ & $0.181 \pm .016(228)$ & $0.251 \pm .015^{\mathrm{b}}(204)$ & $0.245 \pm .006^{\mathrm{b}}(204)$ \\
\hline Season of Birth & $* *$ & $* *$ & $\mathrm{NS}$ & $* *$ \\
\hline Summer(3-5) & $0.316 \pm .016^{\mathrm{a}}(127)$ & $0.169 \pm .019^{\mathrm{a}}(120)$ & $0.296 \pm .021(98)$ & $0.238 \pm .009^{\mathrm{a}}(98)$ \\
\hline $\begin{array}{c}\text { Pre- } \\
\text { Monsoon(6-8) }\end{array}$ & $0.321 \pm .015^{\mathrm{a}}(194)$ & $0.256 \pm .019^{\mathrm{b}}(187)$ & $0.248 \pm .021(161)$ & $0.253 \pm .009^{\mathrm{a}}(161)$ \\
\hline Monsoon $(9-11)$ & $0.260 \pm .010^{\mathrm{b}}(332)$ & $0.277 \pm .013^{\mathrm{b}}(266)$ & $0.285 \pm .015(186)$ & $0.275 \pm .006^{\mathrm{b}}(186)$ \\
\hline Winter(12-2) & $0.104 \pm .027^{\mathrm{c}}(33)$ & $0.079 \pm .034^{\mathrm{a}}(28)$ & - & - \\
\hline Period of Birth & $* *$ & $\mathrm{NS}$ & $\mathrm{NS}$ & $* *$ \\
\hline $2002-2005$ & $0.226 \pm .011^{\mathrm{a}}(419)$ & $0.208 \pm .014(380)$ & $0.295 \pm .016(261)$ & $0.241 \pm .006^{\mathrm{a}}(261)$ \\
\hline $2006-2011$ & $0.275 \pm .015^{\mathrm{b}}(267)$ & $0.182 \pm .019(221)$ & $0.257 \pm .018(184)$ & $0.269 \pm .007^{\mathrm{b}}(184)$ \\
\hline
\end{tabular}

NS - not significant ; * significant $(\mathrm{P}<0.05)$; ** significant $(\mathrm{P}<0.01)$.

Means with different superscripts under each effect within the column differ significantly.

Table 5: Full-sib estimates of heritability and genetic correlation along with standard error for body-weight at several ages (weeks) of crossbred pigs (using REML method of estimation)

\begin{tabular}{|c|c|c|c|c|c|c|c|c|c|c|c|c|c|c|c|c|}
\hline $\begin{array}{l}\text { We } \\
\text { ek }\end{array}$ & 0 & 1 & 2 & 3 & 4 & 5 & 6 & 7 & 8 & 12 & 16 & 20 & 24 & 28 & 32 & 36 \\
\hline 0 & $\begin{array}{c}\mathbf{0 . 7 1} \\
\mathbf{8} \\
(0.00 \\
9)\end{array}$ & $\begin{array}{c}0.30 \\
8 \\
(.012 \\
)\end{array}$ & $\begin{array}{c}0.38 \\
6 \\
(.011 \\
2)\end{array}$ & $\begin{array}{c}0.39 \\
3 \\
(.010 \\
)\end{array}$ & $\begin{array}{c}0.40 \\
6 \\
(.011 \\
)\end{array}$ & $\begin{array}{c}0.29 \\
3 \\
(.011 \\
)\end{array}$ & $\begin{array}{c}0.22 \\
9 \\
(.011 \\
)\end{array}$ & $\begin{array}{c}0.23 \\
0 \\
(.012 \\
)\end{array}$ & $\begin{array}{c}0.29 \\
7 \\
(.008 \\
)\end{array}$ & $\begin{array}{c}\mathbf{0 . 4 2} \\
\mathbf{2} \\
(.011 \\
)\end{array}$ & $\begin{array}{c}0.36 \\
3 \\
(.014 \\
)\end{array}$ & $\begin{array}{c}0.41 \\
4 \\
(.014 \\
)\end{array}$ & $\begin{array}{c}0.36 \\
6 \\
(.016 \\
)\end{array}$ & $\begin{array}{c}0.36 \\
5 \\
(.016 \\
)\end{array}$ & $\begin{array}{c}0.35 \\
5 \\
(.02 \\
0)\end{array}$ & $\begin{array}{c}0.22 \\
8 \\
(.031 \\
)\end{array}$ \\
\hline 1 & $\begin{array}{c}0.42 \\
7\end{array}$ & $\begin{array}{c}0.83 \\
4 \\
(0.01 \\
5)\end{array}$ & $\begin{array}{c}0.80 \\
9 \\
(.005 \\
)\end{array}$ & $\begin{array}{c}0.62 \\
8 \\
(.008 \\
)\end{array}$ & $\begin{array}{c}0.54 \\
3 \\
(.009 \\
)\end{array}$ & $\begin{array}{c}0.37 \\
6 \\
(.011 \\
)\end{array}$ & $\begin{array}{c}0.34 \\
4 \\
(.011 \\
)\end{array}$ & $\begin{array}{c}0.25 \\
7 \\
(.012 \\
)\end{array}$ & $\begin{array}{c}0.11 \\
5 \\
(.013 \\
)\end{array}$ & $\begin{array}{c}- \\
0.10 \\
6 \\
(.022 \\
)\end{array}$ & $\begin{array}{c}- \\
0.05 \\
8 \\
(.024 \\
)\end{array}$ & $\begin{array}{c}0.00 \\
5 \\
(.027 \\
)\end{array}$ & $\begin{array}{c}0.05 \\
2 \\
(.030 \\
)\end{array}$ & $\begin{array}{c}0.07 \\
8 \\
(.031 \\
)\end{array}$ & $\begin{array}{c}0.00 \\
4 \\
(.03 \\
7)\end{array}$ & $\begin{array}{c}0.40 \\
3 \\
(.050 \\
)\end{array}$ \\
\hline 2 & $\begin{array}{c}.0 .38 \\
3\end{array}$ & $\begin{array}{c}0.73 \\
5\end{array}$ & $\begin{array}{c}0.68 \\
3 \\
(0.01 \\
\end{array}$ & $\begin{array}{c}0.88 \\
0 \\
(.003\end{array}$ & $\begin{array}{c}0.82 \\
4 \\
(.004\end{array}$ & $\begin{array}{c}0.61 \\
8 \\
(.008\end{array}$ & $\begin{array}{c}0.61 \\
9 \\
.008\end{array}$ & $\begin{array}{c}0.57 \\
8 \\
(.009\end{array}$ & $\begin{array}{c}0.47 \\
2 \\
(.010\end{array}$ & $\begin{array}{c}- \\
0.03 \\
3\end{array}$ & $\begin{array}{c}0.02 \\
2 \\
(.025\end{array}$ & $\begin{array}{c}0.08 \\
5 \\
(.027\end{array}$ & $\begin{array}{c}0.11 \\
6 \\
(.030\end{array}$ & $\begin{array}{c}0.20 \\
5 \\
(.030\end{array}$ & $\begin{array}{c}0.07 \\
5 \\
(.03 \\
\end{array}$ & $\begin{array}{c}0.44 \\
4 \\
(.048 \\
\end{array}$ \\
\hline
\end{tabular}




\begin{tabular}{|c|c|c|c|c|c|c|c|c|c|c|c|c|c|c|c|c|}
\hline & & & 2) & ) & ) & ) & ) & ) & ) & $\begin{array}{c}(.026 \\
)\end{array}$ & ) & ) & ) & ) & 6) & ) \\
\hline 3 & $\begin{array}{c}0.38 \\
1\end{array}$ & $\begin{array}{c}0.61 \\
1\end{array}$ & $\begin{array}{c}0.83 \\
0\end{array}$ & $\begin{array}{c}0.69 \\
1 \\
(0.01 \\
1)\end{array}$ & $\begin{array}{c}0.87 \\
8 \\
(.003 \\
)\end{array}$ & $\begin{array}{c}0.70 \\
5 \\
(.006 \\
)\end{array}$ & $\begin{array}{c}0.66 \\
4 \\
(.007 \\
)\end{array}$ & $\begin{array}{c}0.56 \\
2 \\
(.008 \\
)\end{array}$ & $\begin{array}{c}0.46 \\
7 \\
(.009 \\
)\end{array}$ & $\begin{array}{c}0.20 \\
0 \\
(.019 \\
)\end{array}$ & $\begin{array}{c}0.19 \\
4 \\
(.021 \\
)\end{array}$ & $\begin{array}{c}0.22 \\
6 \\
(.022 \\
)\end{array}$ & $\begin{array}{c}0.15 \\
4 \\
(.027 \\
)\end{array}$ & $\begin{array}{c}0.17 \\
5 \\
(.029 \\
)\end{array}$ & $\begin{array}{c}0.04 \\
8 \\
(.03 \\
6)\end{array}$ & $\begin{array}{c}0.38 \\
8 \\
(.050 \\
)\end{array}$ \\
\hline 4 & $\begin{array}{c}0.33 \\
2\end{array}$ & $\begin{array}{c}0.52 \\
2\end{array}$ & $\begin{array}{c}0.73 \\
3\end{array}$ & $\begin{array}{c}0.85 \\
1\end{array}$ & $\begin{array}{c}0.57 \\
7 \\
(0.01 \\
1)\end{array}$ & $\begin{array}{c}0.91 \\
1 \\
(.002 \\
)\end{array}$ & $\begin{array}{c}0.81 \\
5 \\
(.004 \\
)\end{array}$ & $\begin{array}{c}0.73 \\
9 \\
(.006 \\
)\end{array}$ & $\begin{array}{c}0.57 \\
8 \\
(.009 \\
)\end{array}$ & $\begin{array}{c}0.40 \\
8 \\
(.019 \\
)\end{array}$ & $\begin{array}{c}0.27 \\
7 \\
(.022 \\
)\end{array}$ & $\begin{array}{c}0.21 \\
9 \\
(.026 \\
)\end{array}$ & $\begin{array}{c}0.06 \\
9 \\
(.031 \\
)\end{array}$ & $\begin{array}{c}0.11 \\
6 \\
(.032 \\
)\end{array}$ & $\begin{array}{c}0.03 \\
3 \\
(.03 \\
7)\end{array}$ & $\begin{array}{c}0.37 \\
0 \\
(.053 \\
)\end{array}$ \\
\hline 5 & $\begin{array}{c}0.29 \\
1\end{array}$ & $\begin{array}{c}0.43 \\
5\end{array}$ & $\begin{array}{c}0.62 \\
4\end{array}$ & $\begin{array}{c}0.75 \\
2\end{array}$ & $\begin{array}{c}0.87 \\
9\end{array}$ & $\begin{array}{c}0.59 \\
7 \\
(0.01 \\
0)\end{array}$ & $\begin{array}{c}0.91 \\
0 \\
(.002 \\
)\end{array}$ & $\begin{array}{c}0.84 \\
2 \\
(.003 \\
)\end{array}$ & $\begin{array}{c}0.71 \\
1 \\
(.006 \\
)\end{array}$ & $\begin{array}{c}0.43 \\
3 \\
(.015 \\
)\end{array}$ & $\begin{array}{c}0.29 \\
3 \\
(.020 \\
)\end{array}$ & $\begin{array}{c}0.26 \\
5 \\
(.023 \\
)\end{array}$ & $\begin{array}{c}0.05 \\
5 \\
(.027 \\
)\end{array}$ & $\begin{array}{c}0.05 \\
1 \\
(.029 \\
)\end{array}$ & $\begin{array}{c}0.03 \\
5 \\
(.03 \\
6)\end{array}$ & $\begin{array}{c}0.18 \\
5 \\
(.059 \\
)\end{array}$ \\
\hline 6 & $\begin{array}{c}0.26 \\
5\end{array}$ & $\begin{array}{c}0.41 \\
2\end{array}$ & $\begin{array}{c}0.58 \\
7\end{array}$ & $\begin{array}{c}0.67 \\
6\end{array}$ & $\begin{array}{c}0.79 \\
6\end{array}$ & $\begin{array}{c}0.86 \\
8\end{array}$ & $\begin{array}{c}0.59 \\
9 \\
(0.01 \\
0)\end{array}$ & $\begin{array}{c}0.91 \\
5 \\
(.002 \\
)\end{array}$ & $\begin{array}{c}0.77 \\
2 \\
(.005 \\
)\end{array}$ & $\begin{array}{c}0.41 \\
8 \\
(.016 \\
)\end{array}$ & $\begin{array}{c}0.30 \\
2 \\
(.020 \\
)\end{array}$ & $\begin{array}{c}0.29 \\
0 \\
(.024 \\
)\end{array}$ & $\begin{array}{c}0.02 \\
4 \\
(.030 \\
)\end{array}$ & $\begin{array}{c}0.12 \\
1 \\
(.031 \\
)\end{array}$ & $\begin{array}{c}- \\
0.07 \\
1 \\
(.03 \\
6)\end{array}$ & $\begin{array}{c}0.16 \\
8 \\
(.063 \\
)\end{array}$ \\
\hline 7 & $\begin{array}{c}0.25 \\
1\end{array}$ & $\begin{array}{c}0.35 \\
7\end{array}$ & $\begin{array}{c}0.53 \\
0\end{array}$ & $\begin{array}{c}0.61 \\
6\end{array}$ & $\begin{array}{c}0.71 \\
8\end{array}$ & $\begin{array}{c}0.80 \\
4\end{array}$ & $\begin{array}{c}0.88 \\
2\end{array}$ & $\begin{array}{c}0.64 \\
7 \\
(0.01 \\
1)\end{array}$ & $\begin{array}{c}0.86 \\
3 \\
(.003 \\
)\end{array}$ & $\begin{array}{c}0.50 \\
3 \\
(.015 \\
)\end{array}$ & $\begin{array}{c}0.29 \\
8 \\
(.021 \\
)\end{array}$ & $\begin{array}{c}0.27 \\
0 \\
(.024 \\
)\end{array}$ & $\begin{array}{c}0.03 \\
5 \\
(.028 \\
)\end{array}$ & $\begin{array}{c}0.23 \\
5 \\
(.029 \\
)\end{array}$ & $\begin{array}{c}0.00 \\
1 \\
(.03 \\
6)\end{array}$ & $\begin{array}{c}0.20 \\
3 \\
(.056 \\
)\end{array}$ \\
\hline 8 & $\begin{array}{c}0.27 \\
0\end{array}$ & $\begin{array}{c}0.30 \\
0\end{array}$ & $\begin{array}{c}0.46 \\
4\end{array}$ & $\begin{array}{c}0.53 \\
5\end{array}$ & $\begin{array}{c}0.62 \\
5\end{array}$ & $\begin{array}{c}0.68 \\
2\end{array}$ & $\begin{array}{c}0.76 \\
0\end{array}$ & $\begin{array}{c}0.86 \\
1\end{array}$ & $\begin{array}{c}0.63 \\
2 \\
(0.00 \\
8)\end{array}$ & $\begin{array}{c}0.60 \\
9 \\
(.007 \\
)\end{array}$ & $\begin{array}{c}0.39 \\
9 \\
(.013 \\
)\end{array}$ & $\begin{array}{c}0.30 \\
3 \\
(.016 \\
)\end{array}$ & $\begin{array}{c}0.12 \\
2 \\
(.019 \\
)\end{array}$ & $\begin{array}{c}0.15 \\
4 \\
(.019 \\
)\end{array}$ & $\begin{array}{c}0.05 \\
1 \\
(.02 \\
2)\end{array}$ & $\begin{array}{c}0.06 \\
5 \\
(.032 \\
)\end{array}$ \\
\hline 12 & $\begin{array}{c}0.28 \\
2\end{array}$ & $\begin{array}{c}0.02 \\
2\end{array}$ & $\begin{array}{c}0.10 \\
1\end{array}$ & $\begin{array}{c}0.28 \\
1\end{array}$ & $\begin{array}{c}0.37 \\
2\end{array}$ & $\begin{array}{c}0.43 \\
7\end{array}$ & $\begin{array}{c}0.46 \\
0\end{array}$ & $\begin{array}{c}0.53 \\
5\end{array}$ & $\begin{array}{c}0.67 \\
8\end{array}$ & $\begin{array}{c}\mathbf{0 . 8 8} \\
\mathbf{0} \\
(0.01 \\
6)\end{array}$ & $\begin{array}{c}0.91 \\
7 \\
(.002 \\
)\end{array}$ & $\begin{array}{c}0.88 \\
7 \\
(.004 \\
)\end{array}$ & $\begin{array}{c}0.74 \\
4 \\
(.008 \\
)\end{array}$ & $\begin{array}{c}0.79 \\
1 \\
(.007 \\
)\end{array}$ & $\begin{array}{c}0.77 \\
2 \\
(.00 \\
9)\end{array}$ & $\begin{array}{c}\mathbf{0 . 5 5} \\
\mathbf{3} \\
(.024 \\
)\end{array}$ \\
\hline 16 & $\begin{array}{c}0.22 \\
4\end{array}$ & $\begin{array}{c}0.00 \\
6\end{array}$ & $\begin{array}{c}0.10 \\
2\end{array}$ & $\begin{array}{c}0.22 \\
9\end{array}$ & $\begin{array}{c}0.28 \\
0\end{array}$ & $\begin{array}{c}0.30 \\
1\end{array}$ & $\begin{array}{c}0.33 \\
3\end{array}$ & $\begin{array}{c}0.37 \\
2\end{array}$ & $\begin{array}{c}0.48 \\
6\end{array}$ & $\begin{array}{c}0.83 \\
3\end{array}$ & $\begin{array}{c}0.85 \\
1 \\
(0.01 \\
8) \\
\end{array}$ & $\begin{array}{c}0.92 \\
7 \\
(.002 \\
) \\
\end{array}$ & $\begin{array}{c}0.83 \\
8 \\
(.006 \\
) \\
\end{array}$ & $\begin{array}{c}0.85 \\
9 \\
(.005 \\
) \\
\end{array}$ & $\begin{array}{c}0.83 \\
8 \\
(.00 \\
7) \\
\end{array}$ & $\begin{array}{c}0.67 \\
8 \\
(.019 \\
) \\
\end{array}$ \\
\hline 20 & $\begin{array}{c}0.22 \\
1\end{array}$ & $\begin{array}{c}0.01 \\
6\end{array}$ & $\begin{array}{c}0.09 \\
0\end{array}$ & $\begin{array}{c}0.19 \\
8\end{array}$ & $\begin{array}{c}0.20 \\
1\end{array}$ & $\begin{array}{c}0.22 \\
9\end{array}$ & $\begin{array}{c}0.25 \\
0\end{array}$ & $\begin{array}{c}0.27 \\
1\end{array}$ & $\begin{array}{c}0.37 \\
7\end{array}$ & $\begin{array}{c}0.74 \\
4\end{array}$ & $\begin{array}{c}0.85 \\
8\end{array}$ & $\begin{array}{c}0.96 \\
6 \\
(0.02 \\
3)\end{array}$ & $\begin{array}{c}0.95 \\
8 \\
(.002 \\
)\end{array}$ & $\begin{array}{c}0.93 \\
4 \\
(.000 \\
)\end{array}$ & $\begin{array}{c}0.94 \\
1 \\
(.00 \\
3) \\
\end{array}$ & $\begin{array}{c}0.81 \\
2 \\
(.012 \\
)\end{array}$ \\
\hline 24 & $\begin{array}{c}0.15 \\
9\end{array}$ & $\begin{array}{c}0.02 \\
2\end{array}$ & $\begin{array}{c}0.08 \\
2\end{array}$ & $\begin{array}{c}0.16 \\
2\end{array}$ & $\begin{array}{c}0.13 \\
5\end{array}$ & $\begin{array}{c}0.12 \\
5\end{array}$ & $\begin{array}{c}0.15 \\
7\end{array}$ & $\begin{array}{c}0.18 \\
8\end{array}$ & $\begin{array}{c}0.25 \\
8\end{array}$ & $\begin{array}{c}0.58 \\
7\end{array}$ & $\begin{array}{c}0.70 \\
7\end{array}$ & $\begin{array}{c}0.86 \\
8\end{array}$ & $\begin{array}{c}0.90 \\
7 \\
(0.02 \\
4)\end{array}$ & $\begin{array}{c}0.95 \\
2 \\
(.002 \\
)\end{array}$ & $\begin{array}{c}0.94 \\
4 \\
(.00 \\
3)\end{array}$ & $\begin{array}{c}0.91 \\
3 \\
(.006 \\
)\end{array}$ \\
\hline 28 & $\begin{array}{c}0.16 \\
8\end{array}$ & $\begin{array}{c}0.03 \\
7\end{array}$ & $\begin{array}{c}0.11 \\
4\end{array}$ & $\begin{array}{c}0.16 \\
5\end{array}$ & $\begin{array}{c}0.13 \\
4\end{array}$ & $\begin{array}{c}0.11 \\
1\end{array}$ & $\begin{array}{c}0.15 \\
5\end{array}$ & $\begin{array}{c}0.22 \\
3\end{array}$ & $\begin{array}{c}0.20 \\
8\end{array}$ & $\begin{array}{c}0.54 \\
7\end{array}$ & $\begin{array}{c}0.65 \\
7\end{array}$ & $\begin{array}{c}0.79 \\
6\end{array}$ & $\begin{array}{c}0.90 \\
2\end{array}$ & $\begin{array}{c}0.88 \\
9 \\
(0.02 \\
4)\end{array}$ & $\begin{array}{c}0.94 \\
3 \\
(.00 \\
3)\end{array}$ & $\begin{array}{c}0.92 \\
9 \\
(.005 \\
)\end{array}$ \\
\hline 32 & $\begin{array}{c}0.18 \\
8\end{array}$ & $\begin{array}{c}0.00 \\
8\end{array}$ & $\begin{array}{c}0.04 \\
8\end{array}$ & $\begin{array}{c}0.08 \\
5\end{array}$ & $\begin{array}{c}0.07 \\
1\end{array}$ & $\begin{array}{c}0.08 \\
5\end{array}$ & $\begin{array}{c}0.09 \\
5\end{array}$ & $\begin{array}{c}0.15 \\
3\end{array}$ & $\begin{array}{c}0.18 \\
9\end{array}$ & $\begin{array}{c}0.55 \\
0\end{array}$ & $\begin{array}{c}0.60 \\
6\end{array}$ & $\begin{array}{c}0.77 \\
3\end{array}$ & $\begin{array}{c}0.86 \\
1\end{array}$ & $\begin{array}{c}0.91 \\
0\end{array}$ & $\$$ & $\$$ \\
\hline 36 & $\begin{array}{c}0.16 \\
3\end{array}$ & $\begin{array}{c}0.14 \\
8\end{array}$ & $\begin{array}{c}0.18 \\
2\end{array}$ & $\begin{array}{c}0.19 \\
1\end{array}$ & $\begin{array}{c}0.12 \\
7\end{array}$ & $\begin{array}{c}0.07 \\
8\end{array}$ & $\begin{array}{c}0.12 \\
6\end{array}$ & $\begin{array}{c}0.21 \\
1\end{array}$ & $\begin{array}{c}0.19 \\
3\end{array}$ & $\begin{array}{c}0.44 \\
0\end{array}$ & $\begin{array}{c}0.51 \\
2\end{array}$ & $\begin{array}{c}0.66 \\
5\end{array}$ & $\begin{array}{c}0.78 \\
5\end{array}$ & $\begin{array}{c}0.86 \\
4\end{array}$ & $\begin{array}{c}0.90 \\
6\end{array}$ & $\begin{array}{c}0.85 \\
4 \\
(0.04 \\
2)\end{array}$ \\
\hline
\end{tabular}

Diagonal elements represent heritability, above diagonal elements represent genetic correlation and below diagonal elements represent phenotypic correlation

\section{Conclusion}

In crossbred pigs, sex of piglet, genetic group did not show marked influence on all pre-weaning body weight measurements except at birth and weaning. Litter size, parity, season of birth and period of birth showed significant effect on the average body weights during pre-weaning periods. However, for post-weaning periods, except for gender, all other factors had a significant effect on average body weights. Similar pattern was observed even for the average daily gains during the pre and post weaning periods. The estimates of heritability indicate that post-weaning body weights are more heritable than pre-weaning body weights. Estimated genetic correlations among pre-weaning bodyweights were positive and low in magnitude while among post-weaning body weights, genetic correlations were high positive. The genetic correlation between birth weight and $3^{\text {rd }}$ month is 0.422 and between $3^{\text {rd }}$ month and $36^{\text {th }}$ month is 0.553 . The estimates of phenotypic correlation between different ages were positive. The finding suggests that birth weight together with $12^{\text {th }}$ week body weight can be used for selection to bring about increased body weight at $36^{\text {th }}$ week. 


\section{Acknowledgement}

The authors are thankful to Tamil Nadu Veterinary and Animal Sciences University, Chennai, India for providing the research facilities for conducting this study.

\section{References}

[1] Joe Cassady. (2002) Genetic parameters and their uses in swine breeding. Swine genetics. Purdue University Cooperative Extension Service, West Lafayette, IN 47907.

[2] D.S. Falconer, Introduction to Quantitative Genetics (3 ${ }^{\text {rd }}$ edition ELBS/Longman, England. UK, 1994).

[3] A. Robertson, Experimental design in the evaluation of genetic parameters, Biometrics, 15, 1959, $219-226$.

[4] SPSS Inc. Released PASW Statistics for Windows, Version 18.0. 2009, Chicago: SPSS Inc.

[5] J. Sai Prasanna, M. Gnana Prakash, B. Ramesh Gupta, M. Mahender and D. Srinivasa Rao, Factor influencing post weaning growth performance in crossbred pigs, Indian Journal of Animal Research 44 (1), 2010, 9-15.

[6] M.G. Prakash, A. Ravi, B.P. Kumari and D. Srinivas Rao, Reproductive and Productive Performance of Crossbred Pigs, Indian Journal of Animal Sciences 78, 2008, 1291-1297.

[7] P. Pandurangareddy, M. Gnana Prakash, B. Punya Kumari, J. Suresh and Alok Bharthi, Genetic analysis of preweaning body weights in crossbred pigs, Indian Journal of Animal Research 47 (1), 2013, 70 - 74.

[8] D. Deka, R.N. Goswami, D.C. Mili, and S. Saikia, Effect of some non genetic factors on Litter traits in Hampshire Pig, Indian Veterinary Journal 79, 2002, 588-90.

[9] B.P. Kumari, D.S. Rao and A. Ravi, Genetic and non-genetic factors affecting the litter traits in desi and crossbred pigs, Indian Veterinary Journal 85, 2008, 170-172. 